\title{
Caractérisation Hydrologique et Sédimentaire de la Lagune de Nador (Maroc)
}

\author{
Mohammed Idrissi, PhD \\ Zineb El Ouhabi, Msc \\ Larissi Jamila, Msc \\ Abdelaziz Agouzouk, \\ Ismail Bessa, Msc \\ Ahmed Makaoui, PhD \\ Karim Hilmi, PhD \\ Omar Ettahiri, PhD
}

Institut National de Recherche Halieutique, Casablanca, Maroc

Doi:10.19044/esj.2020.v16n27p277 URL:http://dx.doi.org/10.19044/esj.2020.v16n27p277

\section{Résumé}

Une prospection océanographique a été réalisée dans la lagune de Nador, située sur la côte méditerranéenne marocaine, en Juillet 2012 dont l'objectif est l'étude de l'état hydrologique et sédimentaire de la lagune après la mise en place de la nouvelle passe en 2011. La distribution des paramètres physico-chimiques du milieu ont permis la mise en évidence une variabilité spatiale en relation avec la circulation générale dans la lagune et la présence de la station d'épuration sur la rive continentale. L'état sédimentaire observé témoigne de l'intensité de l'échange mer-lagune qui est élevée au centre par comparaison aux extrémités NO et SE. Ainsi, le maintien de l'équilibre environnemental de cet écosystème dépend du degré d'échange mer-lagune et le contrôle de l'impact des activités anthropiques.

Mots clés : Maroc, lagune de Nador, Hydrologie, Sédiment 


\title{
Hydrological and Sedimentary Characterization of the Nador Lagoon (Morocco)
}

\author{
Mohammed Idrissi, PhD \\ Zineb El Ouhabi, Msc \\ Larissi Jamila, Msc \\ Abdelaziz Agouzouk, \\ Ismail Bessa, Msc \\ Ahmed Makaoui, PhD \\ Karim Hilmi, PhD \\ Omar Ettahiri, PhD
}

Institut National de Recherche Halieutique, Casablanca, Maroc

\begin{abstract}
An oceanographic survey was carried out in the Nador lagoon located on the Moroccan Mediterranean coast, in July 2012. The objective is to study the hydrological and sedimentary state of the lagoon after the establishment of the new pass, in 2011. The distribution of the physicochemical parameters of the environment allowed the identification of a spatial variability in relation to the general circulation in the lagoon and the presence of the treatment station on the continental shore. The sedimentary state also shows the intensity of the sea-lagoon exchange which is elevated in the center compared to the NO and SE extremities. Thus, maintaining the environmental balance of this ecosystem depends on the degree of sea-lagoon exchange and the control of the impact of anthropogenic activities.
\end{abstract}

Keywords: Morocco, Nador lagoon, Hydrology, Sediment

\section{I - Introduction}

La lagune de Nador appelée aussi Mar Chica ou Sebkha Bou Areg, est la plus grande lagune du littoral marocain présentant une superficie de 115 $\mathrm{km}^{2}$. Elle est située au Nord-Est du littoral méditerranéen marocain entre le cap des trois fourches et le cap de l'eau. Allongée parallèlement à la côte, cet écosystème se présente en un bassin ovale, séparé de la mer par un cordon dunaire orienté NO - SE occupant une bande côtière de $25 \mathrm{~km}$ de longueur. La lagune communique avec la Méditerranée à travers une passe appelée localement Boukhana et son alimentation hydrique est assuré, à la fois, par les eaux marines, la nappe phréatique et les nombreux émissaires de la bordure 
continentale. Cet écosystème est classé en Site d'Intérêt Biologique et Ecologique (SIBE) depuis 1996 et en site Ramsar de conservation et protection des zones humides depuis 2005.

Vue son importance socio-économique pour la région, la lagune de Nador a fait l'objet de certaines études focalisées essentiellement sur la structure hydrologique, sédimentologique, les activités de pêche et d'aquaculture (Guelorget et al., 1987 ; Lefebvre et al., 1996 ; Malouli, 2003 ; Arid et al., 2005 ; Lakhdar et al., 2005 ; Abdellaoui et al. 2006 et 2015 ; Hamoumi, 2012 ; Mostareh et al., 2016; Aknaf et al., 2015 et 2017). Cependant, les études des processus physiques régissant la lagune de Nador et son fonctionnement hydrodynamique ont été entreprises avant la mise en place de la nouvelle passe (outistonsky et al., 2006, Umgeisser et al., 2005 et 2014 ; Hilmi et al., 2003, 2005, 2015). Toutefois, la faible ouverture de cette lagune sur la mer, l'activité industrielle, la croissance démographique et l'augmentation des besoins de la population humaine sont autant de facteurs qui ont contribué à la mise en place d'une nouvelle passe en 2011, pour permettre un meilleur échange des eaux de la lagune avec les eaux méditerranéennes. Cette nouvelle passe de $300 \mathrm{~m}$ de largeur et $6 \mathrm{~m}$ de profondeur, se situe à 1500m à l'ouest de l'ancienne passe.

L'objectif du présent travail est d'étudier les caractéristiques hydrologiques et sédimentaires de la lagune de Nador et les interactions entre les différentes composantes après l'ouverture de la nouvelle passe. Pour cela, une prospection océanographique a été réalisée en Juin 2012 le long de lagune de Nador. Cette prospection est basée sur l'étude des paramètres physicochimiques (Température, Salinité, Oxygènes dissous, matiere en suspension, $\mathrm{pH}$ et sels nutritifs) et sédimentologiques (granulométrie et matière organique). Cet étude a permis d'observée que la situation de la lagune en Juillet 2012 est caractérisée par un rafraichissement des eaux de toute la lagune.

\section{II - Matériel et Méthodes}

Une prospection océanographique a été réalisée du 25 Juin au 04 Juillet 2012 le long de la lagune de Nador. Le réseau d'échantillonnage est composé de 19 stations couvrant la totalité de la lagune en plus d'une station de référence (St 20) en plein mer près de la nouvelle passe (Fig. 1). 

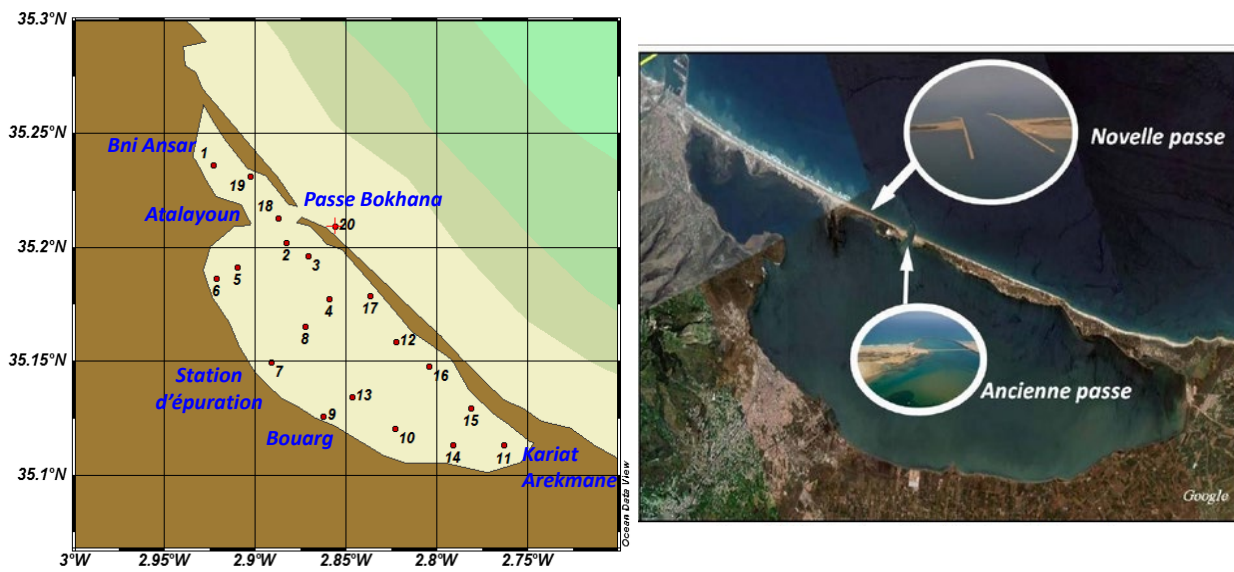

Figure 1 : Réseau des stations océanographiques dans la lagune de Nador (Juin - Juillet 2012) et photo de l'emplacement des passes (Google Earth)

\section{Collecte et traitement des échantillons}

Les paramètres physicochimiques (température, salinité) ont été mesurés sur le terrain à l'aide d'une sonde WTW. Le prélèvement des échantillons d'eau a été effectué en surface et au fond, ainsi :

Les analyses des sels nutritifs (l'azote Ammoniacal (NH4+), les nitrites (NO2), les nitrates (NO3-) et les phosphates (PO4)) ont été réalisées selon les méthodes appropriées (Benshneider et Robinson, 1952 ; Murphy et Riley, 1962 ; Wood et al., 1967 ; Koroleff, 1969) et dosés à l'aide un spectrophotomètre UV-1205.

La matière en suspension (MES) a été obtenue par filtration à vide (filtres de Whatman $\mathrm{GF} / \mathrm{F}$ de diamètre de $47 \mathrm{~mm}$ ).

la concentration de l'oxygène a été obtenue par la méthode de Winkler.

L'analyse du sédiment prélevé le long de la lagune est représentée par la granulométrie et le pourcentage de la matière organique. La texture du substrat est évaluée à partir du pourcentage pondéral après tamisage sous l'eau courante (Plumb, 1981 ; CEAEQ, 2003). L'échelle de classification est celui adoptée par Wenthworth (Foucault et Raoult, 1988). La détermination des faciès des sédiments est faite à partir du positionnement du pourcentage de chaque fraction dans le diagramme triangulaire de Folk (1974 modifié).

La détermination de la matière organique est obtenue par incinération des échantillons (perte de poids au feu ou perte par calcination) (CEAEQ, 2003). On place les échantillons pendant une nuit (16 heures) dans un four à moufle à $375^{\circ} \mathrm{C}$. Le pourcentage de la matière organique est calculé à partir de :

$\%$ M.O. = (poids éch. sec (g) - poids éch. incinéré (g) / poids éch. sec (g)) $\times 100$ 


\section{Traitement des données}

Les résultats relatifs aux différents paramètres mesurés sont présentés sous forme de cartes de distribution spatiale à l'aide du logiciel Ocean Data View (ODV).

\section{III - Résultats :}

\section{Paramètres physico-chimiques}

Les distributions de la température de surface le long de la lagune de Nador, varient entre $23,1^{\circ} \mathrm{C}$ et $29,8^{\circ} \mathrm{C}$ avec une moyenne de $26,7^{\circ} \mathrm{C}$. Les plus faibles valeurs ont été enregistrées près de la passe en contact direct avec l'eau de mer où les eaux entrantes étaient plus fraiches que celles de l'intérieur de la lagune. Par ailleurs, les valeurs élevées ont été enregistrées en amont de la lagune le long de la rive continentale où le maximum était enregistré au niveau de la station $9\left(29,8^{\circ} \mathrm{C}\right)$ (Fig. 2).

En profondeur les températures ont varié entre $22,4^{\circ} \mathrm{C}$ à $29,1^{\circ} \mathrm{C}$ avec une moyenne de $25,7^{\circ} \mathrm{C}$. Les eaux entrantes plus fraiches occupaient presque toute la largeur de la partie centrale de la lagune, alors que les parties amont et aval étaient plus chaudes (Fig. 2).

La variation de distribution des salinités entre la surface et le fond était peu apparente. Les valeurs variaient entre $36,1 \mathrm{psu}$ et $38,3 \mathrm{psu}$ avec une moyenne de $37,3 \mathrm{psu}$. Les distributions étaient presque similaires avec la partie centrale moins saline que les extrémités amont et aval. A l'inverse de la température, la station 9 enregistrait la plus faible salinité de surface $(36,1 \mathrm{psu})$. (Fig. 2).

Concernant les teneurs en oxygène dissous de surface, elles variaient entre 2,34 et $5,22 \mathrm{mg} / 1$. La distribution spatiale montre une concentration des faibles teneurs en amont de la lagune qui faisaient la continuité avec les faibles teneurs entrantes de la mer. Au reste de la lagune, les eaux étaient mieux oxygénées avec une forte teneur en face de la passe, près de la rive continentale (station 5).

En profondeur, les valeurs étaient comprises entre 1,86mg/1 et 4,4mg/1 avec une moyenne de $3,3 \mathrm{mg} / \mathrm{l}$. La distribution spatiale montre une zone limitée de fortes teneurs près de la passe et une zone de faibles teneurs à l'extrémité amont de la lagune (Fig. 2). 

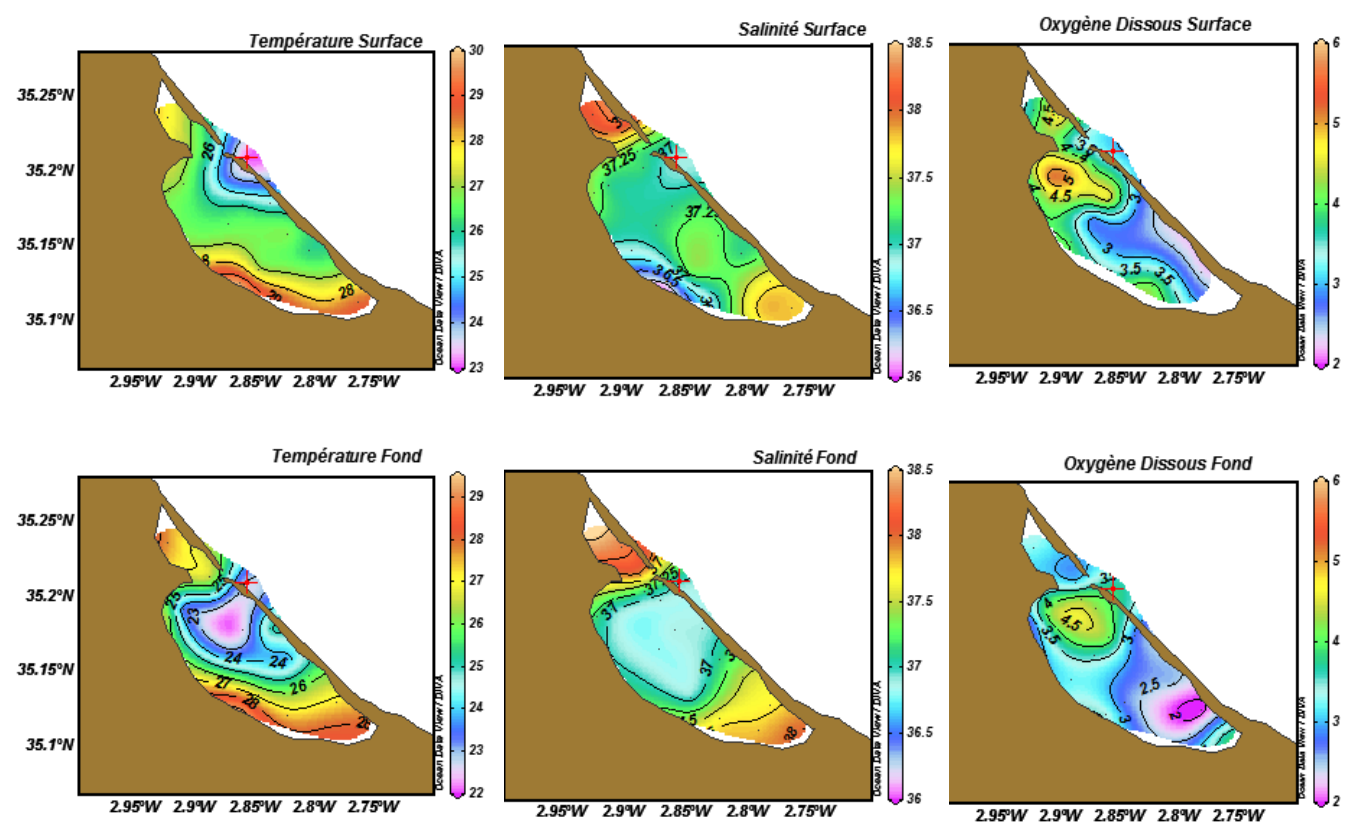

Figure 2 : Distributions en surface et au fond des paramètres hydrologiques dans la lagune de Nador (Juin - Juillet 2012)

Quant au pH, les taux sont assez élevés au niveau de toute la lagune, les eaux de la lagune sont légèrement alcalines, les valeurs varient entre 8,2 et 8,5 (Fig. 3).

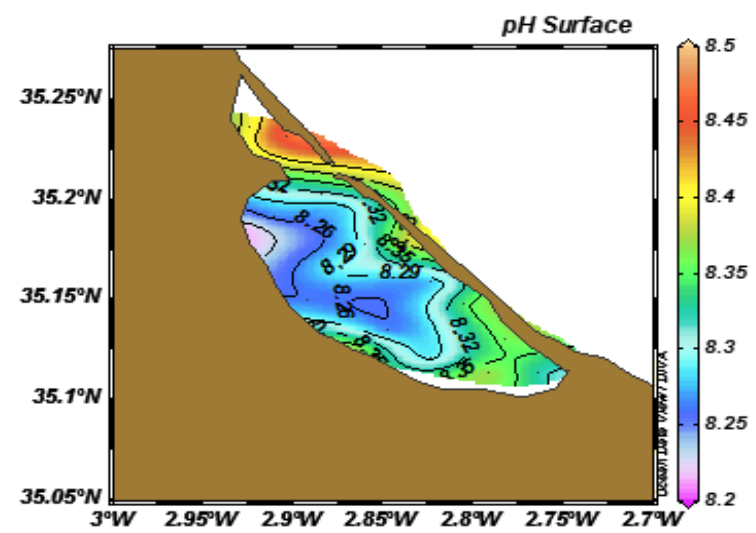

Figure 3 : Distribution en Surface du pH dans la lagune de Nador (Juin - Juillet 2012)

\section{Sels nutritifs}

En ce qui concerne les teneurs en sels nutritifs enregistrées le long de la lagune de Nador en période d'été 2012, différents schémas de distribution spatiale étaient observés entre les différent composants et entre la surface et le fond (Figure 4). Globalement, les distributions spatiales étaient caractérisées par la présence de foyers de fortes concentrations dont l'emplacement est 
variable considérant chaque élément à part. Ainsi, les phosphates et les nitrites étaient plus concentrés près de la passe, en surface, à l'extrémité N-O de la lagune ; alors que les nitrates se concentraient au centre de la lagune sur la rive continentale en surface et à l'extrême partie S-E au fond. Par ailleurs, les fortes teneurs en azote ammoniacal étaient localisées en face de la passe près d'Atalyun aussi bien en surface qu'au fond.
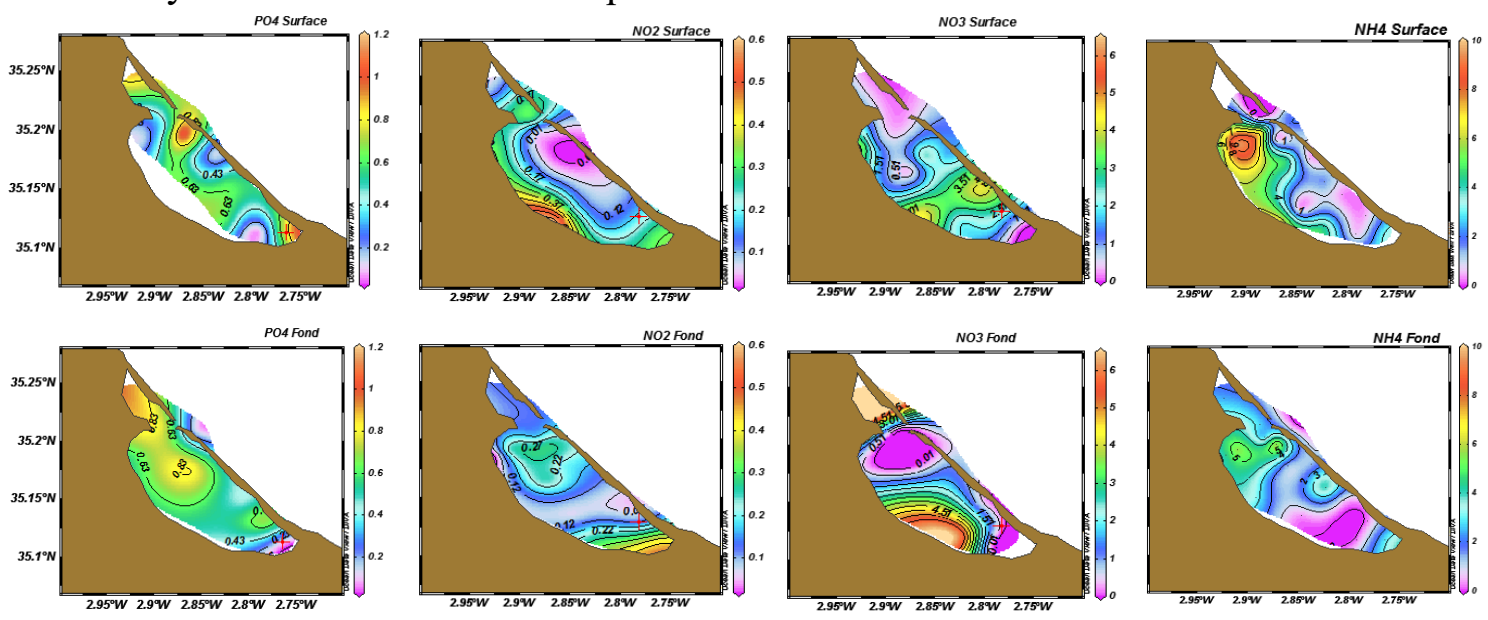

Figure 4 : Distributions des teneurs en sels nutritifs en surface et au fond dans la lagune de Nador (Juin - Juillet 2012)

\section{Matière en suspension}

La turbidité exprimée par le taux de matière en suspension le long de la lagune de Nador était moyenne $(<47 \mathrm{mg} / \mathrm{l})$, ce qui indique une faible action dynamique au sein de la lagune. Néanmoins, la distribution spatiale des taux de la MES a permis une individualisation d'une zone de forte turbidité sur la rive continentale entre Bouaarg et Atalayun où le maximum a été observé au fond, près de la station d'épuration (Fig. 5).
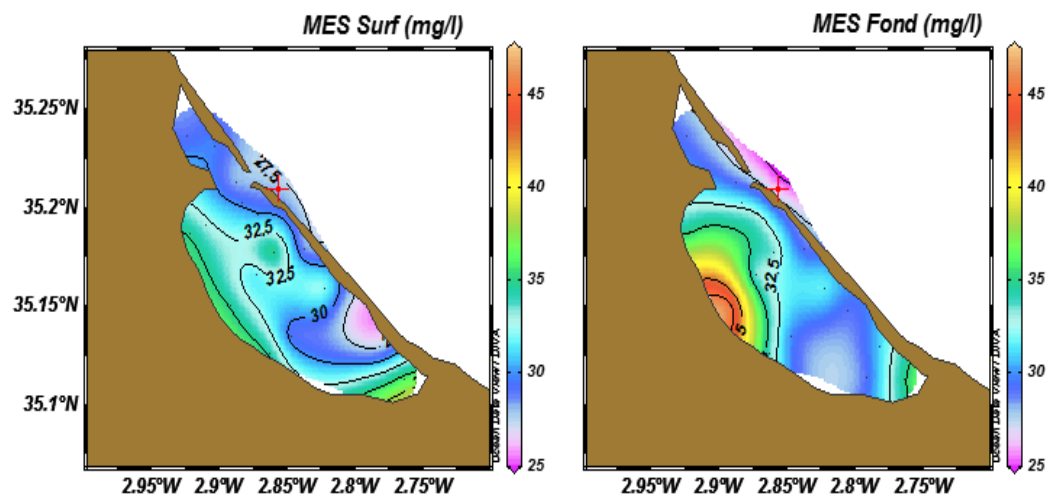

Figure 5 : Distributions de la matière en suspension (MES) en surface et au fond dans la lagune de Nador (Juin - Juillet 2012) 


\section{Sédiments}

L'analyse granulométrique du sédiment de la lagune de Nador, a montré la présence de sable grossier à moyen au niveau de la passe, du chenal principal circulant dans la direction transversale de la lagune et au niveau de la bordure de la lagune adossée au cordon dunaire. Tandis que les deux extrémités de la lagune (Kariat Arkmane et Beni N'sar) étaient caractérisées par un faciès vaseux à vaso-sableux ce qui traduit la faible hydrodynamique de ces deux régions (Figure8).

Par ailleurs, le taux de la matière organique estimé au niveau de la lagune de Nador, fluctuait entre 1 et $15 \%$. La distribution spatiale de cette composante montre un gradient croissant de la passe vers l'intérieur de la lagune et vers les extrémités $\mathrm{S}-\mathrm{O}$ et $\mathrm{N}-\mathrm{E}$ où les teneurs les plus élevées ont été enregistrées. Au niveau de la passe et le milieu de la lagune, les teneurs étaient faibles à cause de la forte agitation en ces zones, ce qui ne permet pas la sédimentation de la fraction fine et, par conséquent, la non sédimentation de la matière organique (Figure 8).
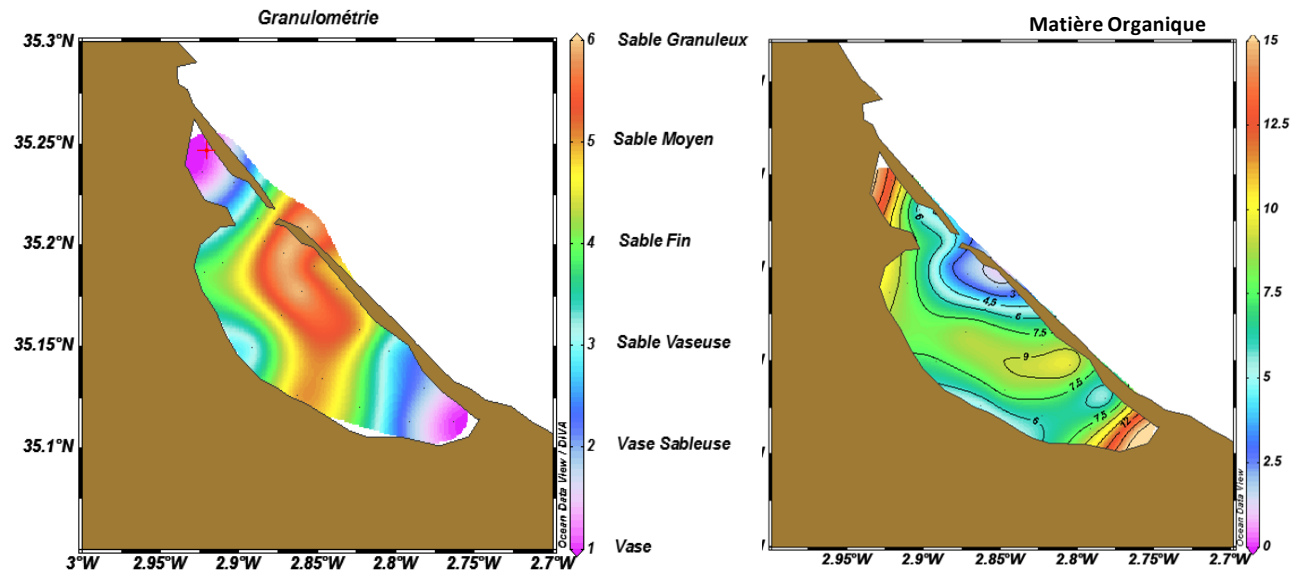

Figure 8 : Distributions en surface de la matière organique et la granulométrie dans la lagune de Nador (Juin - Juillet 2012)

\section{IV- Discussion et Conclusion}

Les caractéristiques hydro-sédimentaires de la lagune de Nador ont été étudiées depuis les années 60 à partir des paramètres hydrologiques et sédimentologiques. Par ailleurs, le risque d'eutrophisation de la lagune a été constaté à partir des années 80 suite au faible contact avec la mer et l'augmentation de l'activité anthropiques sur la bordure continentale de la lagune (Abouhala et al., 1995 ; Inani, 1995 ; Lefebvre et al., 1996 ; Bloundi, 2005).

Concernant les aspects hydrologiques de la lagune de Nador, des études réalisées en Juillet 2002 et 2004, et en Juin 2006 avant l'ouverture de la nouvelle passe, montrent que les eaux les plus fraiches étaient localisées au 
niveau de la passe $\left(22,1\right.$ et $25^{\circ} \mathrm{C}$ respectivement $)$; alors que l'intérieur de la lagune était plus chaud dont le maximum des températures était enregistré sur la bordure continentale à faible profondeur (jusqu'à $30^{\circ} \mathrm{C}$ ) (Abdellaoui et al., 2005 ; Bloundi, 2005 ; Zerrouqi et al., 2013). De même, la salinité enregistrait des valeurs élevées en 2002 et 2004 qui dépassaient 40psu (Bloundi, 2005). Toutefois, la situation observée en Juillet 2012 est caractérisée par un rafraichissement des eaux de toute la lagune avec des teneurs de salinité plus faibles $(36,1$ - 38,3psu). Ce constat prouve un début d'amélioration de la circulation des eaux à l'intérieur de la lagune grâce à la nouvelle passe qui a eu un impact favorable sur la qualité des eaux de cet écosystème. En effet, les teneurs en sels nutritifs enregistrées en Juillet 2012 témoignent d'un d'apport important de ces éléments par comparaison à 2002 et 2004 (Abdellaoui et al., 2005 ; Bloundi, 2005). Cet apport peut être dû aussi bien à une meilleure alimentation de la lagune par l'eau marine et la présence la station d'épuration sur la rive continentale de la lagune. Nos résultats sont comparables à ceux publiés récemment par Aknah et al. (2015), selon une étude réalisée en Juillet 2013. Le schéma inverse est observé pour les distributions de l'oxygène dissous en relation avec la qualité des eaux dans différentes parties de la lagune. Toutefois, les fluctuations du $\mathrm{pH}$ sont faibles dont les marges oscillent entre 8,22 et 8,47 et sont presque similaires aux valeurs trouvées par Lefebvre et al. (1996) et Zerrouqi (2013) ce qui ne montre pas de relation directe de ce paramètre avec les changements hydrologiques du milieu. Le $\mathrm{pH}$ est un paramètre complexe dont la valeur dépend de nombreux facteurs tant chimiques que biologiques ce qui rend les faibles variations difficilement interprétables (Lefebvre et al., 1996).

De même et par référence à l'état sédimentaire dans la lagune, les deux extrémités $\mathrm{NO}$ et $\mathrm{SE}$, sont relativement à l'écart de cette circulation et constituent des zones plus ou moins confinées, naturellement enrichies en matière organique. Ces résultats sont en concordance avec ceux de Abouhala et al. (1995) et Lefebvre et al. (1996) qui estiment que ces deux parties tendent à se différencier du reste de la lagune du fait de leur faible profondeur et de leur éloignement par rapport à la communication mer-lagune ce qui diminue la circulation dans ces zones. De ce fait, le schéma général de la circulation dans la lagune de Nador est toujours maintenu mais avec des degrés variables d'intensité en relation avec la capacité d'échange mer-lagune. Ainsi, depuis l'ouverture de la nouvelle passe en 2011, la lagune de Nador a changé de classification d'une lagune 'étouffée' (choked lagoon) à une lagune 'coulante' (leaky lagoon) permettant un meilleur échange de ses eaux avec la mer Méditerranée (Umgeisser et al., 2014 ; Hilmi et al., 2015).

En conclusion, la lagune de Nador comme tout écosystème littoral manifeste une grande variabilité spatio-temporelle des caractéristiques hydrologiques et sédimentaires qui est tributaire du degré de mélange des eaux 
douces avec les eaux marines et de l'action anthropiques. En conséquence, le maintien de l'équilibre de cet écosystème nécessite l'amélioration de son hydrodynamisme par le maintien d'un bon niveau de la communication merlagune et la protection de la qualité de ses eaux par la mise en place de systèmes d'épuration efficaces.

\section{References:}

1. Adellaoui B., Nhhala B. et Talbaoui E.M. (2015) Mediterranean coastal lagoons: sustainable management and interactions among aquaculture, Capture fisheries and the environment: Studies and Reviews. General Fisheries Commission for the Mediterranean, 95, FAO: 163-173. ISBN 978-92-5-108792-3.

2. Abdellaoui B., Najih M. et Idhalla M. (2005). Fonctionnement hydrologique et risques d'eutrophisation de la lagune de Nador. Rapport scientifique lagmar, 10p.

3. Abouhala A., Boukabous R., Dafir J., Talbaoui M., (1995). Caractérisation physico-chimique de la lagune de Nador. Actes Inst. Agron. Vet., 15(4): 43-52.

4. Aknaf A., Akodad M., Moumen A., Ben Chekroun K., Elhamouti C., Bailal A., Baghour M., (2015). Impact of the new pass on the eutrophication of the lagoon Marchica: Study of the two sites Bou Areg and Mohandis. J. Mater. Environ. Sci., 6(10) : 2939-2943

5. Aknaf A., M. Akodad, M. Layachi, F. El Madani, A. Jaddar, A. Mesfioui, M. Baghour (2017). Study of the spatial and temporal variation of physical-chemical parameters characterizing the quality of surface waters of the lagoon Marchica - North-East Morocco. Journal of Materials and Environmental Sciences ISSN: 2028-2508. JMES, Volume 8, Issue 9, Page 3216-3225.

6. Arid H., Moudni, H. Orbi, A., Talbaoui, E.M., Lakhdar, I.J., Massik, Z., Littaye, A. et Paoli, R. (2005). Télédétection spatiale et SIG pour la gestion intégrée des potentialités aquacoles. GEO OBSERVATEUR. Centre Royal de Télédétection Spatiale, $\mathrm{N}^{\circ} 14$, pp 63-80.

7. Bendschneider K. et Robinson R. J. (1952). A new spectrophotometric method for the determination of nitrite in sea water. J. Mar. Res. 11 : 87-96.

8. Bloundi M.K. (2005). Etude géochimique de la lagune de Nador (Maroc oriental) : Impacts des facteurs anthropiques. Thèse de Doctorat Université Mohammed V, Rabat et ULP-EOST Strasbourg I, $215 p$.

9. CEAEQ (2003). Determination of Organic Matter by Determination of Organic Carbon in Agricultural Soils by Method of Walkley-Black's 
Modified Method. MA. 1010-WB 1.0. Ministry of Environment of Quebec.

10. Folk, R. L. (1974). Petrology of Sedimentary Rock, Hemphill Publishing Company, Austin, TX.

11. Foucault, Raoult (1988). Dictionnaire de géologie, Paris 1988, [3 éd.].

12. Guelorget O. et Perthuisot J.P. (1983). Le domaine paralique. Expressions géologiques, biologiques et économiques du confinement. Trav. Lab. Geol, ENS, Paris, 16, 136p.

13. Guelorget O., Perthuisot, J.P., Frisoni, G.F. et Monti, D. (1987). Le rôle du confinement dans l'organisation biogéologique de la lagune de Nador (Maroc). Oceanologica Acta, $10: 435-444$.

14. Hamoumi N. (2012). Le complexe lagunaire de Nador (Maroc): fonctionnement, contrôle naturel et provoqué, scenarii d'évolution future. Revue Paralia, Volume 5, pp 5.1-5.14.

15. Hilmi, K., Koutitonsky, V.G., et Orbi, A. (2003). Circulation et dispersion tridimensionnelle (3D) de la lagune de Nador durant l'automne 2001 (septembre-novembre 2001). Travaux et Documents INRH, 112, $47 \mathrm{p}$.

16. Hilmi K. (2005). Modélisation numérique de la circulation de deux milieux paraliques du littoral marocain : la lagune de Oualidia (Atlantique) et la lagune de Nador (Méditerranée). Thèse de Doctorat, Université Hassan II-Mohammedia, 186 p.

17. Hilmi K., Koutitonsky, V.G., Orbi A., and M. Chagdali, M. (2005). Three dimensional water circulation and dispersion in Nador lagoon (Morocco). In: P. Lasserre, P. Viarioli and P. Campostrini Eds: Lagoons and Coastal Wetlands, in the Global Change Context: Impacts and Management Issues, Proceedings of the International Conference, Venice, 26-28 April 2004, ICAM Dossier $N^{\circ} 3$, UNESCO.

18. Hilmi K., Makaoui A, Idrissi M., Abdellaoui B., El Ouehabi Z. (2015). Circulation marine de la lagune de Nador (Maroc) par modélisation hydrodynamique. European Scientific Journal, 11(32): 418-428.

19. Koroleff F. (1969). Direct determination of ammonia in natural waters as indophenol blue. ICES, C. M. / C: 9

20. Koutitonsky V.G, Orbi, A., Hilmi, K. and Berraho Ab. (2006). Benefits of a proposed second inlet on water renewal in Nador lagoon, Morocco. The $1^{\text {st }}$ International Conference: Environmental Change in lakes, lagoons \& wetlands of the southern Mediterranean region, 4-7 january 2006, Cairo, Egypt.

21. Lakhdar Idrissi J., Orbi A., Hilmi K., Zidane F. et Moncef M. (2005). Modèles de température et de la qualité de l'eau appliquée à deux lagunes marocaines : Oualidia (Atlantique) et Nador (Méditerranée). Environnemental Technology, 26 : 713-720. 
22. Lefebvre A., Guelorget O., Perthuisot J.P., Dafir J.E. (1996). Evolution biogéologique de la lagune de Nador (Maroc) au cours de la période 1982 1993. Oceanologica Acta, 20(2) : 371-385.

23. Malouli Idrissi M., Zahri Y., Houssa R., Abdellaoui B., et Elouamari N. (2003). Pêche artisanale dans la lagune de Nador : Exploitation et aspects socio-économiques. Informes y Estudios, 10 ; COPEMED : 61122.

24. Mostareh Mohammed, Faid ELmadani, Hameed Saleh Ali Yahya, Ouafae EL Hachemi, Souad Abdellaoui, Kamal Abbas Merghem, Abdelhafid Chafi (2016). Physico-chemical assessment of the Nador lagoon's water quality - North of the Eastern Morocco - after the opening of the new inlet. J. Mater. Environ. Sci. 7 (12), 4795-5809 Mostarih et al. ISSN: 2028-2508 CODEN: JMESCN.

25. Murphy J. and Riley J.P. (1962). A modified single solution method for the determination of phosphate in natural waters. Anal. Chim. Acta, $27: 31-36$.

26. Plumb, R.H. 1981. Procedure for handling and chemical analysis of sediment and water samples. Tech. Rep. EPA/CE-81/1. U.S. Army Engineer Waterways Experiment Station, Vicksburg. MS, 71pp.

27. Umgiesser G., Chao J., Bajo M., Scroccaro I., Cucco A. (2005). Residence time modelling in the Nador lagoon, Morocco. In Proceedings of the First International Conference on Coastal Conservation and Management in the Atlantic and Mediterranean (ICCCM'05), F. Veloso Gomes, F. Traveira Pinto, L. das Neves, A. Sena and O. Ferreira (eds), pp. 389-397.

28. Umgiesser G., Ferrarin C., Cucco A., De Pascalis F., Bellafiore D., Ghezzo M., Bajo M. (2014). Comparative hydrodynamics of 10 Mediterranean lagoons by means of numerical modeling. J. Geophys. Res. Oceans, 119(221): 26 pages.

29. Wood E.D., Armstrong F.A.J. and Richards F.A. (1967). Determination of nitrate in sea water by cadmiumCopper reduction to nitrite. J. Mar. Biol. Ass. U. K. 47: 23-31.

30. Zerrouqi Z., Sbaa M., Chafi A. et Aqil H., (2013). Contribution à l'étude de la qualité des eaux de la lagune de Nador : Impact de l'anthropisation. Bulletin de l'Institut Scientifique, Rabat, Section Sciences de la Vie, 35 : 51-59. 